\title{
Bandwidth and gain enhancement of a circular microstrip antenna using a DNG split ring resonator radome
}

\author{
Ojo Rasheed ${ }^{1}$, Mohd Faizal Jamlos ${ }^{2}$, Ping Jack $\mathrm{Soh}^{3}$, Mohd Aminudin Jamlos ${ }^{4}$, Muammar \\ Mohamad Isa ${ }^{5}$ \\ ${ }^{1,3}$ Advanced Communication Engineering Centre (ACE), School of Computer and Communication Engineering, \\ Universiti Malaysia Perlis, Malaysia \\ ${ }^{2}$ Faculty of Mechanical Engineering, Universiti Malaysia Pahang, 26600, Pekan, Malaysia \\ ${ }^{4}$ Department of Electronic, Faculty of Technology Engineering, Universiti Malaysia Perlis, Malaysia \\ ${ }^{5}$ School of Microelectronic Engineering, Universiti Malaysia Perlis, Malaysia
}

\section{Article Info}

Article history:

Received Dec 12, 2018

Revised Jan 25, 2019

Accepted Feb 14, 2019

\section{Keywords:}

Circular patch

Double negative (DNG)

Metamaterial

Radome

Split ring resonator

\begin{abstract}
This paper present the design of a circular patch microstrip antenna with enhancement in terms of bandwidth and gain using a dielectric double negative (DNG) split ring metamaterial radome. This radome is positioned on top of the CP antenna operating from $5.2 \mathrm{GHz}$ to $6.4 \mathrm{GHz}$. The metamaterial radome comprises of two alternate split rings of negative permittivity, permeability and refractive index. The circular microstrip antenna bandwidth of $430 \mathrm{MHz}$ has been realized by the presence of DNG metamaterial radome compared to $220 \mathrm{MHz}$ without the radome. The gain has been increased as well from $1.84 \mathrm{dBi}$ to $3.87 \mathrm{dBi}$.
\end{abstract}

Copyright () 2019 Institute of Advanced Engineering and Science. All rights reserved.

\section{Corresponding Author:}

Mohd Faizal Jamlos,

Faculty of Mechanical Engineering, Universiti Malaysia Pahang,

26600, Pekan, Malaysia.

Email: mohdfaizaljamlos@gmail.com

\section{INTRODUCTION}

The enhancement of microstrip antennas using radomes enhances their practical application, besides protecting them from different environmental effects such as wind, rain, ice, ultraviolet radiation etc. Radome can be of different shapes such as spherical, geodesic, planar etc., dependings on the application (radar, telemetry, tracking, and point-to-point). They can be designed using different types of low losses materials, for example_fiberglass, and coated using PTFE- for indoor and outdoor environments [1], However, the main challenge for designing radome is avoiding antenna degradation in terms of attenuation, scattering and depolarization. Furthermore, scattered radiation from the use of radomes may degrade antenna performance in terms of antenna directivity via the enhancement of side lobes [2], The main disadvantages of the microstrip patch antenna are narrower band and low gain [3], Due to these factors, many researches have investigated methods to improve the bandwidth and antenna gain by modifying the ground plane orradiating patch [4-5].

A different types of artificial magnetic conductor has been used to design radome as-partially reflecting surfaces, or frequency selective surfaces. Metamaterial-based radomes can be great addition to microstrip antennas due to the unusual electromagnetic properties that they are able to provide [6-7], Metamaterial attains exotic electromagnetic property over certain frequency band, which is abnormal in nature. It can show totally opposite characteristics compared to natural materials in terms of negative permittivity or permeability [8], An axial symmetrical CRLH circular patch antenna was proposed to support the zeroth order mode metamaterial [9], MNZ or MNG metamaterial in the central part of circular patch 
would increase the resonant frequency of mode TM11 while keeping the frequency of mode TM21 unchanged, and there would be increment of bandwidth by enlarged if they are so close to connect two band together [10], A compact low-profile dual-band patch antenna in which designed by inserting an array of the TL-MTM unit cell into the conventional microstrip patch. a compact single-layer dual-band patch antenna design. The TL-MTM unit cell has advantages for antenna design such as small size and aperture enhancement [11], the using ENZ MTM as a dielectric substrate remarkably improved the antenna impedance bandwidth. Such super-bandwidth phenomenon is demonstrated experimentally by exploiting the intrinsic dispersion characteristic of metallic wires inspired in a dielectric substrate [12], TL metamaterial is derived to analyze this circular antenna. Since the unit cells of this TL can reinforce unpredictable phase shift, the antenna can form negative modes, positive modes and zeroth-order resonant (ZOR) modes. For a ZOR antenna, the resonant frequency is independent of its physical size. The resonant frequency, input impedance and radiation characteristics of the ZOR circular antenna are analyzed in terms of the physical parameters of the unit cells [13], a compact triple-band microstrip antenna based on MTM complementary split ring resonator (CSRR) with two different geometries loaded on the ground plane of the antenna. MTM lens that is created by a modified H-shape unit cells. A MTM metamaterial super lens positioned at the focal point above the patch is introduced using a novel complementary $\mathrm{H}$-shaped unit cells to maximize the gain and mounted on the overall antenna structure.

This work explores the design of such metamaterial split, ring radome for a circular patch antenna operating at $5.8 \mathrm{GHz}$. A method is used to extract the effective permeability, refractive index, and impedance from S-parameter to analyze whether the metamaterial structure is also DNG at the same frequency.

However, another advantages of using Double Negative (DNG) Split Ring Resonator (SRR) radome. Some research are going on to improve the bandwidth and gain of patch antenna. Existing solutions leads to the problems of specious radiation and high complexity. This new approach come up with a new solution called metamaterial radome, which play an important role in the antenna design due to its interesting and unusual properties.

\section{ANTENNA DESIGN AND UNIT CELL RADOME}

Explaining In this proposed work, a circular patch microstrip antenna resonating at $5.8 \mathrm{GHz}$ designed. The crucial inconvenience of a microstrip antenna is its narrow bandwidth. Thus, Double Negative (DNG) Split Ring Resonator (SRR) radome is designed to focus on enhancing the bandwidth and gain by achieving the maximum efficiency. Circular patch antenna is chosen since due to the less complexity compared to rectangular patch antenna since it has only one parameter to control i.e. radius. Circular patch antenna ha`s been designed based on (1):

Circular Patch Radius

$$
\alpha=\frac{F}{\left\{1+\frac{2 h}{\pi F \varepsilon_{r}}\left[\ln \left(\frac{\pi F}{2 h}\right)+1.7726\right]\right\} 1 / 2}
$$

Where;

$$
\mathrm{F}=\frac{8.791 \times 10^{9}}{f_{r} \sqrt{\varepsilon_{r}}}
$$

The complete structure of proposed antenna radome is etched using FR4 substrate $\varepsilon \mathrm{r}=4.3$ with dimension of $56 \times 56 \mathrm{~mm}$, and a dielectric thickness of $=1.6 \mathrm{~mm}$. Figure 1(a) shows the perspective view of the circular patch antenna with radius of the patch denotes as ' $a$ ' while thickness of the dielectric substrate notifies by ' $h$ '. Figure 1(b) and (c) show the front and back view of circular patch microstrip antenna respectively. Figure 2(a) presents the DNG-SRR structure where the permittivity and permeability is in negative scale ranging from 2 to $8 \mathrm{GHz}$. Figure 2(b) illustrates the complete simulation setup of proposed structure where the circular antenna has been covered by the DNG-SRRs which act as radome. The detailed dimensions of the proposed structure is given in Table 1. 


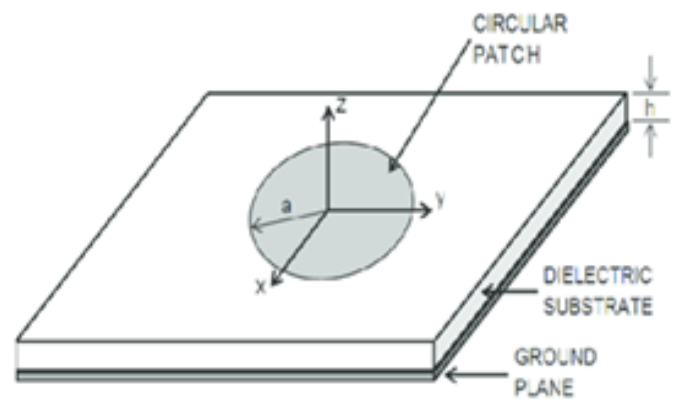

(a)

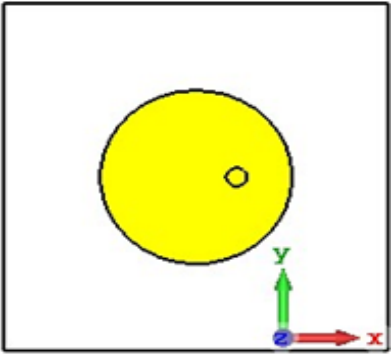

(b)

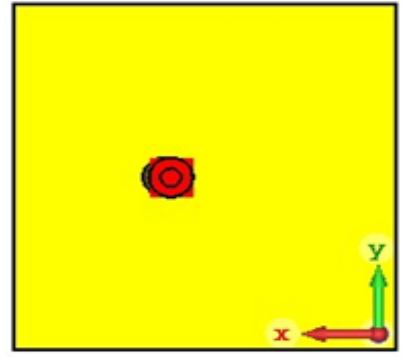

(c)

Figure 1. Design of circular patch antenna, (a) perspective view, (b) front view, (c) back view

Table 1. The specification of proposed antenna and radome

\begin{tabular}{cccc}
\hline Parameters & Unit $(\mathrm{mm})$ & Parameters & Unit $(\mathrm{mm})$ \\
\hline L & 56 & $\mathrm{~L}$ & 4.4 \\
W & 56 & $\mathrm{~W}$ & 12.5 \\
Radius CP & 5.9 & $\mathrm{~b}$ & 0.27 \\
Frequency & $5.8 \mathrm{GHz}$ & $\mathrm{w}$ & 3.9 \\
Sub. Material & FR4 & $\mathrm{g}$ & 0.3 \\
Permittivity. & $\varepsilon_{\mathrm{r}}=4.3$ & & \\
Sub. Thick. & 1.6 & & \\
\hline
\end{tabular}

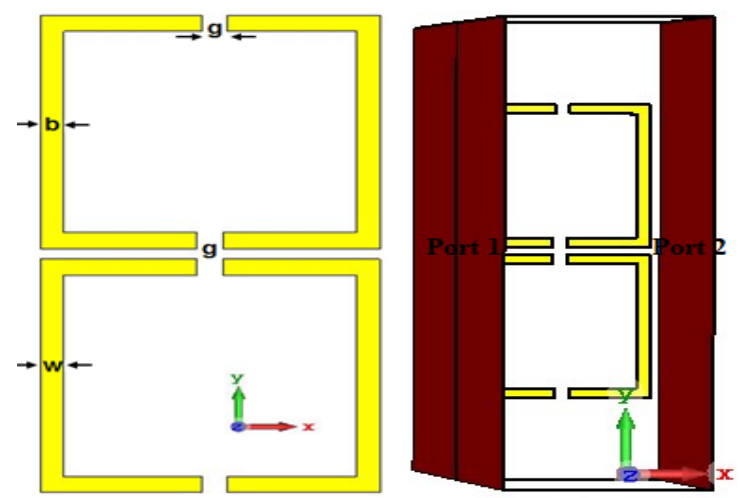

(a)

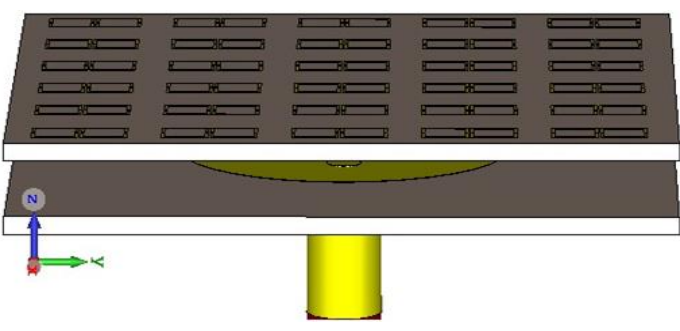

(b)

Figure 2. Proposed antenna structure, (a) DNG-SRR unit cell, (b) DNG-SRRs located on top of circular patch antenna

The negative permittivity and permeability of the structure that are obtained from the simulated complex value of S-parameters of Transmission Reflection (TR) technique of electromagnetic radiation at the boundary between two surfaces. Next, Nicolson-Ross-Weir (NRW) technique is deployed to find negative 
values of permittivity and permeability [14]. The negative values of permittivity and permeability are retrieved by exporting the scattering parameters (S11, and S21) to MATLAB using the following equations [15-16],

$$
\begin{aligned}
& \mathcal{E}=n / \mathrm{z} \\
& \mu=n \mathrm{z}
\end{aligned}
$$

where

$$
\begin{aligned}
& n=\frac{1}{k d} \cos ^{-1}\left[\frac{1}{2 S_{21}}\left(1-S_{11}^{2}+S_{21}^{2}\right)\right] \\
& \mathrm{z}=\sqrt{\frac{\left(1+S_{11}\right)^{2}-S_{21}^{2}}{\left(1+S_{11}\right)^{2}-S_{21}^{2}}}
\end{aligned}
$$

\section{RESULTS AND ANALYSIS}

In this section, it is explained the results of research and at the same time is given the comprehensive discussion. Results can be presented in graphs, DNG-SRR radome structure with the circular patch antenna is simulated using CST Microwave Studio electromagnetic simulator. Figure 3 (a)-(d) present the relative permittivity, permeability, refractive index and impedance of the unit cell of DNG-SRR. This unit cell array have been applied on top of circular patch antenna in $5 \mathrm{~mm}$ distance.

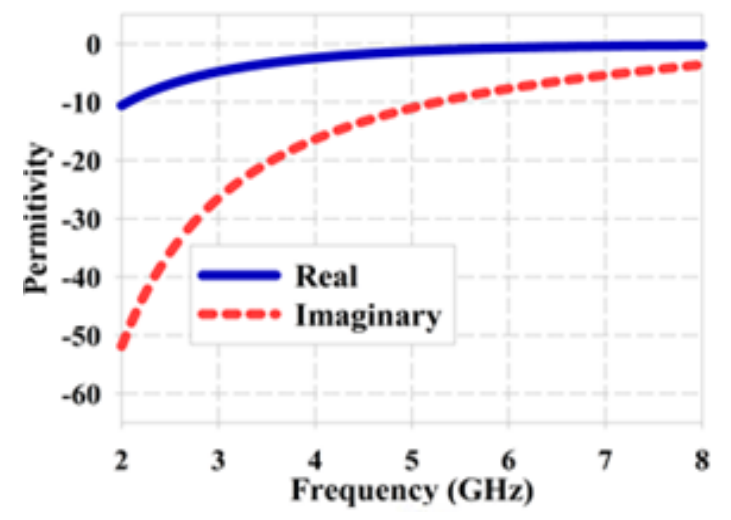

(a)

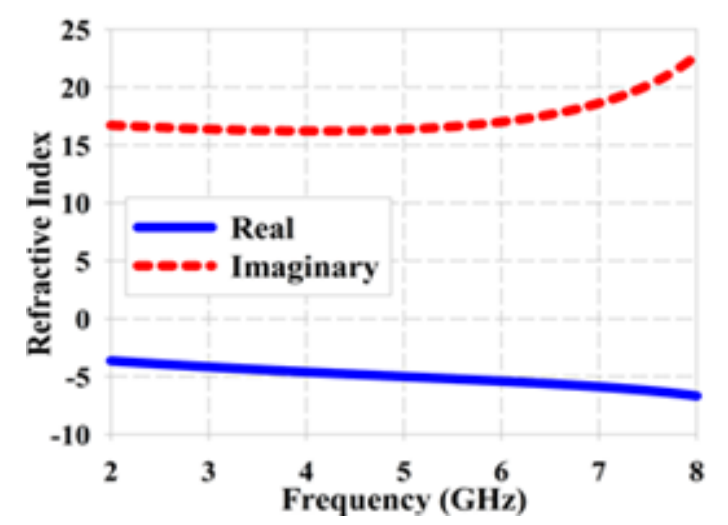

(c)

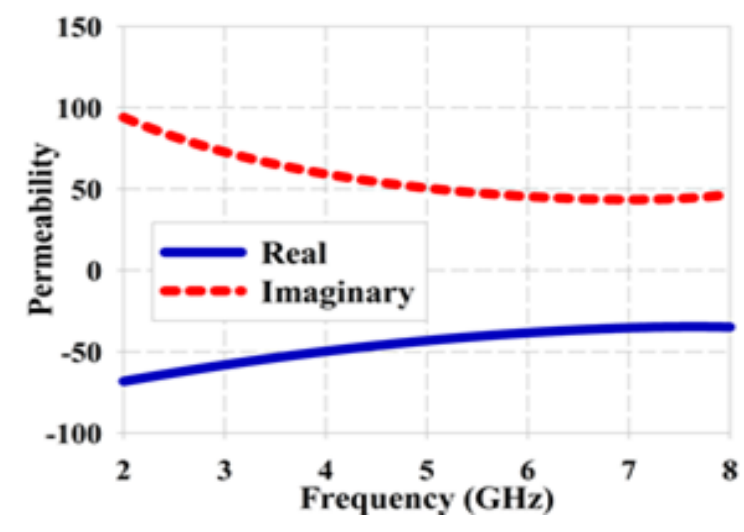

(b)

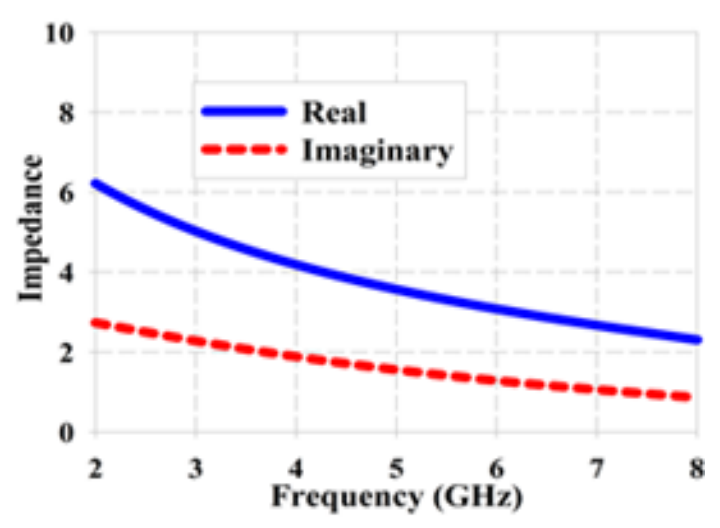

(d)

Figure 3. Characterization of DNG-SRR unit cell, (a) Permittivity, (b) Permeability, (c) Refractive index, (d) Impedance 
Figure 3(a) and (b) proven that the SRR-based unit cell of SRR is a double negative (DNG) unit cell since both negative permittivity and negative permeability are stated in a negative Y-axis for both parameters.These figures give substantiation results of a negative behavior of the periodic structure, which simulation of negative permittivity and permeability shows good compliance. Meanwhile negative refractive index and positive impedance is shown in Figure 3(c) and (d). The resulting bandwidth and gain of the final antenna is illustrated in Figure 4 and 5 respectively. The enhancement of bandwidth is observed to be 210 $\mathrm{MHz}$ while gain is observed to be $3.9 \mathrm{~dB}$ at $5.8 \mathrm{GHz}$.

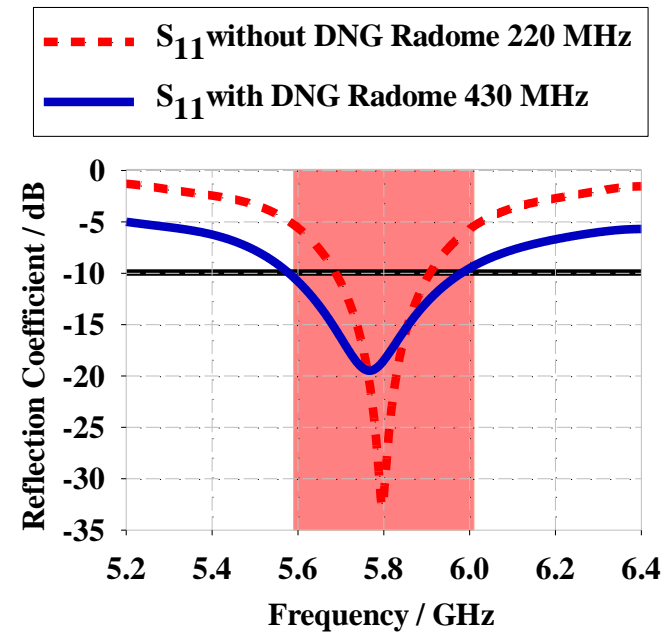

Figure 4. Effects of DNG-SRRs S11 comparison between with and without radome

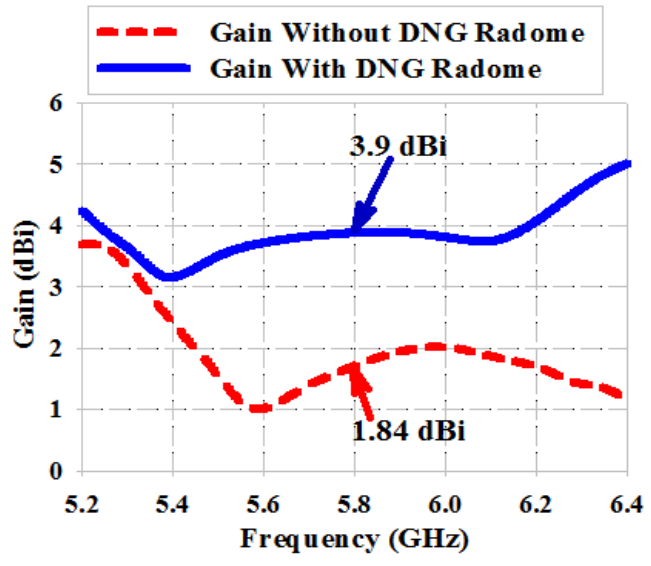

Figure 5. The effects of Gain comparison between with and without radome

\section{CONCLUSION}

Provide The paper concludes that DNG-SRRs could increase the antenna gain and bandwidth, which could be useful for antenna radome applications. The features of negative permeability, permittivity and refractive index, in which produced using MATLAB from Transmission Reflection (T/R) technique using direct retrieval method from simulated S11 and S21 is contributed by the DNG-SRRs, which placed on the top of circular patch microstrip antenna. The S11 shows a $7.43 \%$ of bandwidth with DNG metamaterial radome. Gain of $3.9 \mathrm{~dB}$ has been realized by the presence of DNG-SRRs radome compared to $1.84 \mathrm{~dB}$ of without the radome. 


\section{ACKNOWLEDGEMENTS}

This work has been partly supported by the Malaysia Ministry of Education under FRGS/1/2017/STG02/UNIMAP/02/2 and UniMAP Research Incentive Fund of 9007-00141. The authors also acknowledge Advanced Communication Engineering Centre (ACE) for the lab facilities.

\section{REFERENCES}

[1] S. Gupta and S. Kumar, "Design and Analysis of Compact and Broadband High Gain Micro strip Patch Antennas," 2014 International Conference on Communication and Network Technologies, Sivakasi, 2014, pp. 11-15.

[2] Joshi, J. G., Shyam S. Pattnaik, and S. Devi. "Metamaterial embedded wearable rectangular microstrip patch antenna." International Journal of Antennas and Propagation 2012 (2012)

[3] A. R. Parvathy and T. Mathew, "A novel aperture coupled microstrip circular patch antenna for dual band operation," 2017 Progress in Electromagnetics Research Symposium - Fall (PIERS - FALL), Singapore, 2017, pp. 1738-1742.

[4] Mondal, Kalyan, et al. "Broadband microstrip patch antenna of reduced sized for multiband applications." Indian Journal of Pure \& Applied Physics (IJPAP) 53.8 (2015): 553-556.

[5] Mondal, Kalyan, and Partha Pratim Sarkar. "Single feed aperture coupled circular broadband microstrip patch antenna." In Devices for Integrated Circuit (DevIC) IEEE, 2017, pp. 737-741., 2017.

[6] Zheng, K-S., et al. "Designing and measurement of a single layered planar gain-enhanced antenna radome with metamaterials." Journal of Electromagnetic Waves and Applications 26.4 (2012): 436-445.

[7] El Alami, Ali, et al. "Modeling the Radiation Characteristics of a Circular Patch Antenna by using Cavity Model for RFID Applications." European Journal of Scientific Research 110.1 (2013): 199-207.

[8] C. Zhang, J. Gong, Y. Li and Y. Wang, "Zeroth-Order-Mode Circular Microstrip Antenna With Patch-Like Radiation Pattern," in IEEE Antennas and Wireless Propagation Letters, vol. 17, no. 3, pp. 446-449, March 2018.

[9] Yang, Kejian, and Zhan Zhang. "High Gain and Wideband Circular Patch Antenna Loaded with MNZ Metamaterials." 2018 International Conference on Microwave and Millimeter Wave Technology (ICMMT) IEEE., 2018.

[10] Zong, Binfeng, et al. "Compact low-profile dual-band patch antenna using novel TL-MTM structures." IEEE Antennas Wireless Propag. Lett. 14 (2015): 567-570.

[11] A. Jafargholi and M. H. Mazaheri, "Broadband microstrip antenna using epsilon near zero metamaterials," in IET Microwaves, Antennas \& Propagation, vol. 9, no. 14, pp. 1612-1617, 19112015.

[12] Yan, Sen, and Guy AE Vandenbosch. "Zeroth-order resonant circular patch antenna based on periodic structures." IET Microwaves, Antennas \& Propagation 8.15 (2014): 1432-1439.

[13] E. K. I. Hamad, W. A. E. Ali, M. Z. M. Hamdalla and M. A. Bassiuny, "High gain triple band microstrip antenna based on metamaterial super lens for wireless communication applications," 2018 International Conference on Innovative Trends in Computer Engineering (ITCE), Aswan, 2018, pp. 197-204.

[14] Hasan, Md Mehedi, Mohammad Rashed Iqbal Faruque, and Mohammad Tariqul Islam. "Compact Left-Handed Meta-Atom for S-, C-and Ku-Band Application." Applied Sciences 7.10 (2017): 1071.

[15] Vicente, Alexandre Natã, Gustavo Maciulis Dip, and Cynthia Junqueira. "The step by step development of NRW method." Microwave \& Optoelectronics Conference (IMOC), 2011 SBMO/IEEE MTT-S International. IEEE, 2011.

[16] Smith, D. R., et al. "Electromagnetic parameter retrieval from inhomogeneous metamaterials." Physical review E 71.3 (2005): 036617

\section{BIOGRAPHIES OF AUTHORS}

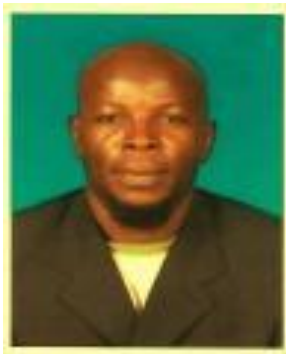

Ojo Rasheed is a Ph.D. student at University Malaysia Perlis- School of Computer and Communication Engineering. His research interests include Antenna Design Systems and Metamaterial Radome, and M.Sc. in 2014 from Universiti Malaysia Perlis, School of Computer and Communication Engineering, and He received the B.Sc. in 2013 Information Technology from Al- Medina International University Shaa Alam Malaysia. 

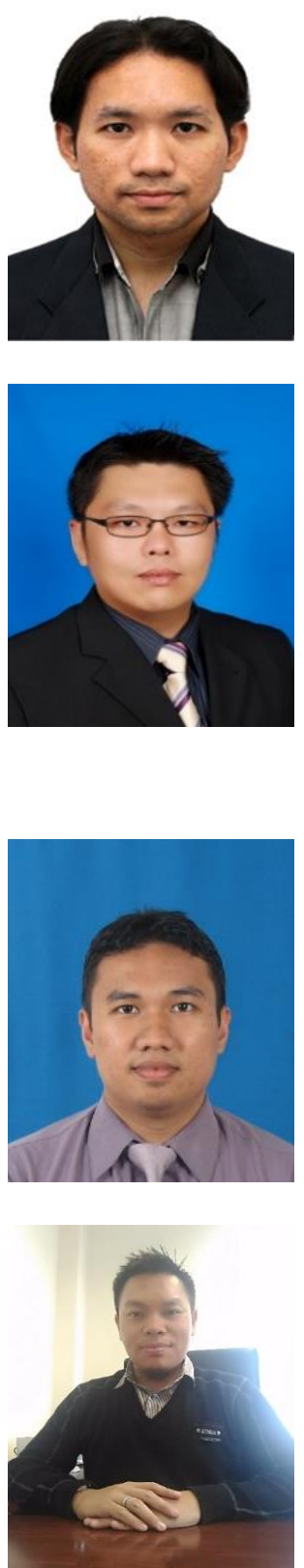

Mohd Faizal Jamlos received Ph.D. in 2010 from Universiti Teknologi Malaysia, Johor, Malaysia and M.Sc. in 2008 from University of Adelaide, South Australia, Australia. He is currently Professor at Faculty of Mechanical Engineering, Universiti Malaysia Pahang (UMP). Previously he was Associate Professor at Advanced Communication Engineering Centre (ACE), School of Computer and Communication Engineering, Universiti Malaysia Perlis. He has co-authored more than 220 scientific publications in peer-reviewed journals and conferences. His research interest are wireless embedded system, remote sensing, on-platform antennas and microwave circuitry and IoT applications. He is a practice professional Engineer of Board of Engineers Malaysia (BEM), Senior Member of IEEE, a National Medical Researcher (NMRR) and Corporate Member of Institute Engineers Malaysia (MIEM).

Ping Jack Soh (S '10, M '14, SM '15) was born in Sabah, Malaysia. He received the B.Eng. and M.Eng. degrees in Electrical Engineering (Telecommunication) from Universiti Teknologi Malaysia (UTM) in 2002 and 2006, respectively, and the PhD degree in Electrical Engineering from KU Leuven, Belgium in 2013. He is currently an Associate Professor at the School of Computer and Communication Engineering (SCCE), Universiti Malaysia Perlis (UniMAP), and also a Research Affiliate at KU Leuven, Belgium. Dr. Soh was the recipient of the IEEE Antennas and Propagation Society (AP-S) Doctoral Research Award in 2012, the IEEE Microwave Theory and Techniques Society (MTT-S) Graduate Fellowship for Medical Applications in 2013 and the International Union of Radio Science (URSI) Young Scientist Award in 2015. He is a Chartered Engineer registered with the UK Engineering Council; a Senior Member of the IEEE, a Member of the IET, ACES and URSI; and Graduate Members of the Board of Engineers Malaysia (BEM) and the Institution of Engineers Malaysia (IEM).

Mohd Aminudin Jamlos received his Doctorate in Communication Engineering from Universiti Malaysia Perlis. Currently, he works as a Senior Lecturer at Engineering Technology Faculty of Engineering Technology, UniMAP. From 2011 to August 2018, he has produced 21 journals and 25 conference papers. Additionally, he also received 9 Gold and 1 Special Award (SIIF 2011) including national and international exhibitions. He is a professional certified in Mikrotik Certified Network Association (MTCNA) and Mikrotik Certified Routing Engineer (MTCRE) in international recognized engineering networks too. He obtained one copyright and one patent pending for research product Alzheimer Early Detection while doing a master's degree. He is interested in doing research in microwave imaging, disease and cancer identification, dielectric material characterization, electromagnetic waves, antennas and microwave measurements.

Muammar Mohamad Isa received his B. Eng. (Hons.) Electrical \& Electronic Engineering from Universiti Tenaga Nasional in 2002 before he joined Silterra (M) Sdn. Bhd. as a Process Engineer. Later, he pursued his M. Sc (Microelectronics) at Universiti Kebangsaan Malaysia in 2004 before he joined Kolej University Kejuruteraan Utara Malaysia (KUKUM) as a full-time academician. After his three years of experience as Lecturer there, he pursued his $\mathrm{PhD}$ in Electrical \& Electronic Engineering at The University of Manchester and received his degree in 2012. His works in the development of high-frequency and low noise devices for satellite communication have been recognized by ANGKASA in 2012. He currently works on high-speed and low-noise device fabrication and characterization for future high-speed, high-frequency and low-noise applications. He also works on the design and fabrication of micro-antenna for early cancer cell detection. He can be contacted at muammar@unimap.edu.my. 